\title{
Fanificación y conectividad en las sociedades
}

hipermediatizadas

Un estudio de caso seleccionado de la plataforma YouTube ${ }^{1}$

Fanification and connectivity in hypermediated societies

A case study selected from the YouTube platform

Fanificação e conectividade em

sociedades hipermediadas

Um estudo de caso seleccionado da plataforma YouTube

\section{FEDERICO ÁLVAREZGANDOLFI}

federicoalvarezg@gmail.com - Consejo Nacional de Investigaciones Científicas y Técnicas, Argentina.

ORCID: https://orcid.org/0000-0002-0860-412X

CÓMO CITAR: Álvarez Gandolfi, F. (2021). Fanificación y conectividad en las sociedades hipermediatizadas. Un estudio de caso de la plataforma YouTube.InMediacionesdela Comunicación, 16(2), 181-206. DOI: https://www.doi.org/10.18861/ ic.2021.16.2.3155
Fecha de recepción: 28 de marzo de 2021

Fecha de aceptación: 18 de junio de 2021

\section{RESUMEN}

Este artículo examina las transformaciones de las mediatizaciones y de las condiciones de circulación del sentido en el marco de las plataformas digitales con base en Internet. Para eso se pone atención en un caso de estudio particular: la fanificación que atraviesa la vinculación entre obras audiovisuales, interacciones, producciones discursivas y prácticas en YouTube relacionadas con el consumo de objetos de la cultura de masas japonesa. El objetivo de este trabajo es poner en

1 El trabajo se realizó con el financiamiento de una beca doctoral otorgada por el Consejo Nacional de Investigaciones Científicas y Técnicas (CONICET), Argentina. 
diálogo las reflexiones sobre tales cambios asociados a la expansión de los medios conectivos y de las lógicas algorítimicas de la dataficación social, con especial énfasis en los interrogantes generados por los estudios sobre fans-campo de constitución incipiente en el ámbito dela academialatinoamericana-. En el contexto de las sociedades contemporáneas hipermediatizadas, se revisa la extrapolación de ciertos postulados en torno de la democracia y la descentralización de las redes sociales, para el análisis de los usos que hacen de YouTube los fans de bienes culturales japoneses, usualmente conceptualizados en términos de prosumidores. Así se contrastan las oportunidades y las limitaciones de la arquitectura de dicha plataforma para la participación, sus apropiaciones y las dinámicas colectivas específicas en torno de uno delosyoutubers o creadores de contenido más populares entre los mencionados fans.

PALABRAS CLAVE: culturas fan, colectivos de identificación, enunciadores hipermediáticos, comunicación digital, YouTube.

\section{ABSTRACT}

This article examines the transformations of mediatizations and the conditions of circulation of meaning on Internet-based digital platforms, centering on a particular case study: that of fanification around audiovisual works, interactions, discursive productions and practices on YouTube related to the consumption of Japanese mass culture. It aims to put into dialogue the reflections on such changes, associated with the expansion of connective media (Van Dijck, 2016) and the algorithmic logic of social datafication (Livingstone, 2018), with the questions inscribed in the field of fan studies, of incipient constitution in the Latin American academy. Indeed, in the context of contemporary hypermediatized societies (Carlón, 2017), this work reviews the extrapolation of certain postulates around democracy and decentralization of social networks for the analysis of the uses that fans of Japanesegoods make of YouTube, usually conceptualized in terms of prosumers. Thus, it contrasts the opportunities and limitations of the architecture of this platform for participation in general, its appropriations and the specific collective dynamics around one of the most popular content creators among these fans.

KEYWORDS: fans cultures, collective identification, hypermedia enunciation, digital communication, YouTube.

\section{RESUMO}

Este artigo examina as transformações das midiatizações edas condições de circulação de sentido no quadro das plataformas digitais baseadas na Internet. Para tanto, foca-se a atenção num estudo de caso particular: o da fanificação que cruza o elo entre obras audiovisuais, interações, produções discursivas e práticas no YouTube relacionadas ao consumo de objetos da cultura de massa japonesa. O objetivo deste trabalho éfazer dialogarem as reflexões sobre tais mudanças associadas à expansão das mídias conectivas e das lógicas algorítmicas da dataficação social, com ênfase especial nas questões geradas pelos estudos sobre fãs -campo de constituição incipiente no âmbito da academia latino-americana-. No contexto das sociedades contemporâneas hipermediatizadas, pretende- se rever a extrapolação de certos pressupostos sobre democracia e descentralização das redes sociais para a análise de usos feitos do YouTube pelos fãs dos bens culturais japoneses, geralmente conceituados em termos de prossumidores. Assim, contrastam-se as oportunidades e limitações da arquitetura dessa plataforma para a participação, suas apropriações e as dinâmicas coletivas específicas em torno de um dos YouTubers ou criadores de conteúdo mais populares entre os referidos fãs.

PALAVRAS-CHAVE: culturas fãs, coletivos de identificação, enunciadores hipermídiaticos, comunicação digital, YouTube. 


\section{INTRODUCCIÓN}

Las reflexiones que aquí se presentan sobre las manifestaciones en YouTube del fanatismo por objetos de la cultura de masas japonesa, principalmente animé y manga -es decir, animaciones e historietas niponas- se inscriben en el marco de una apuesta general por problematizar los modos en que suele comprenderse el panorama mediático contemporáneo y sus implicaciones socioculturales. Frente a las transformaciones propias del contexto actual de la cultura de la convergencia (Jenkins, 2008) de los medios de comunicación masiva, tradicionales y digitales, ya es indiscutible la consolidación de la posibilidad de que los receptores puedan ser también emisores y visibilizar sus discursos en un grado mayor al que permitía el sistema broadcasting, clásico modelo de emisión centralizada o de transmisión punto-masa (Carlón \& Scolari, 2009).

En este sentido, se alcanzó cierto consenso respecto del papel de las audiencias como usuarias de plataformas y redes en Internet o, en otros términos, respecto de que los consumidores pueden profundizar la actividad implicada en toda interpretación situada, creando contenidos propios de modo colaborativo y produciendo textos que comparten en la web (Hills, 2013). De allí que es fundamental para nuestros intereses integrar-en el análisis- el desarrollo de las plataformas digitales, las cuales otorgan mayor notoriedad a las prácticas de losfans, y leer la convergencia mediática en su dimensión material y simbólica como un proceso tecnológico y sociocultural.

Sobre la base del consenso antes mencionado, otra noción usualmente aplicada -junto con la de convergencia- para dar cuenta del papel activo de las audiencias en general, y de los fans en particular, es la de cultura participativa (Jenkins, 2009a, 2013). De este modo, hay quienes afirman que los cambios generados por la digitalización habilitan otros modos de participación y colaboración de las audiencias convertidas en usuarios, tanto como el flujo más libre de ideas y contenidos, pues "la convergencia es tanto un proceso corporativo de arriba abajo como un proceso de abajo arriba dirigido por los consumidores" (Jenkins, 2008, p. 28). A partir de Internet, no solo se asiste a una revolución del acceso (Verón, 2013) de los actores individuales a la discursividad mediática, sino también a cambios de las relaciones de los individuos con el conocimiento y la cultura, de las relaciones interindividuales y de las relaciones entre los individuos y las instituciones.

No obstante, si bien se habla mucho de la participación a la hora de referir a las "nuevas" actividades delas audiencias en el marco de una cultura colaborativa y de la convergencia, se hacen pocas menciones a cómo se materializa dicha participación a partir de los medios. Más bien se la engloba como ejemplo de la riqueza y la refuncionalización implicadas en la creatividad fan (Booth, 2015). 
En tal sentido, se hace necesario reconocer las continuidades históricas entre las características mediáticas y las prácticas de consumo antes y después de la consolidación de Internet (Jenkins, 2009b), también es fundamental precisar las formas específicas que cobra la convergencia y los modos particulares en los que se despliegan las actividades participativas, más allá de los aspectos democratizadores, descentralizados o empoderadores que suelen, a priori, adjudicárseles (Bollmer, 2015; Jenkins, boyd \& Ito, 2016).

Uno de los problemas consiste en que no necesariamente todos los consumidores de medios se comprometen con el contexto de convergencia y participación, más allá de que aumenten sus posibilidades de hacerlo, así como tampoco todos sus discursos cobran el mismo valor o logran el mismo reconocimiento (Couldry \& Hay, 2011). De allí que no debería presuponerse la consolidación o generalización del paradigma del llamado prosumidor (Scolari, 2008) y evitar, de ese modo, caer en el riesgo de cierto determinismo tecnológico en función del cual termine por entenderse que las plataformas digitales son en sí mismas responsables de nuevas formas horizontales de ordenamiento social y manifestación cultural. Las negociaciones, tensiones, contradicciones y desigualdades siguen atravesando tales formas de uso e intercambio, lo cual puede ilustrarse mediante la reflexión sobre los contenidos, las apropiaciones, los significados y los vínculos que se generan en YouTube entre los fans de productos mediáticos japoneses, conocidos como otakus, ya que el crecimiento de este colectivo no solo involucra dinámicas de identificación entre pares, sino también de distinción interna.

Cabe subrayar que una de las dimensiones en las cuales reside la relevancia analítica de este colectivo de fans es el aumento de su visibilidad, motivada justamente por la digitalización y el incremento de la popularidad del animé y el manga a nivel mundial (Jenkins, boyd \& Ito, 2016). En Iberoamérica, por caso, el acceso de los otakus a estos productos se realiza mayoritariamente a través de Internet, con traducciones al español oficiales o hechas por fans para fans. Entre los indicios que hablan de un creciente mega-nicho de mercado se encuentran, por ejemplo, los números de las distribuidoras tras el lanzamiento de ciertos títulos, su presencia cada vez mayor en plataformas como Netflix y hasta el lanzamiento de sitios de streaming específicos como Crunchyroll. Asimismo, en lo que hace a la conceptualización particular de este fandom, colectivo o comunidad de fans que comparten gustos e intereses -en este caso asociados a bienes simbólicos japoneses-, los otakus suelen ser pensados como un ejemplo de funcionamiento tanto horizontal como colaborativo y promotores de acciones creativas alternativas (Eng, 2012). Además, tienden a ser relacionados con una valoración positiva de la diversidad cultural, ya que no solo disfrutan de las animaciones e historietas niponas, sino que también les atrae la sociedad y la cultura de Japón en general (Jenkins, 2009a). 
En ese marco, resulta entonces fundamental-a la hora del análisis- matizar ciertos supuestos generalizadores que reposan en la celebración acrítica de los usos de las plataformas digitales, cayendo muchas veces en un determinismo tecnológico que no permite complejizar los alcances de la reflexión (Benítez Larghi et al., 2014). Pero esta matización tampoco debe caer en una suerte de miserabilismo tecnológico. Lo que debe revisarse es la supuesta potencialidad igualadora de las redes sociales y la supuesta horizontalidad de plataformas como YouTube, que serían capaces de promover creaciones contrahegemónicas (Hidalgo-Marí \& Segarra-Saavedra, 2017; Márquez \& Ardèvol, 2018). Tales caracterizaciones tienden a no contemplar la reflexión sobre las jerarquías intracomunitarias y las prácticas que reproducen lógicas dominantes dentro de los colectivos estudiados.

A continuación, se analizarán las posibilidades y los límites de la puesta en circulación en YouTube de contenidos y discursos vinculados a las prácticas de consumo de objetos de la cultura de masas japonesa. Con esa finalidad, se realizará una sistematización de los distintos niveles de participación que habilita la plataforma y sus posibles usos, en pos de complejizar su conceptualización como espacio de circulación de prosumos. A partir de esa primera exploración, se reflexionará sobre las apropiaciones, haciendo hincapié en uno de los youtubers -entendidos en términos generales como nuevos sujetos mediáticos (Scolari \& Fraticelli, 2017)- más populares entrelos fans delos bienes culturales nipones: Kalathras.

El corpus sobre el cual se desarrollará el análisis comunicacional y sociocultural propuesto está construido de acuerdo con criterios cuantitativos que establecen la "popularidad" de los videos y canales en YouTube (Burgess \& Green, 2018), es decir, su representatividad medida a partir del número de visualizaciones, suscriptores, reacciones y comentarios. Tal representatividad fue contrastada con aproximaciones cualitativas, y sostenida en un doble trabajo de campo etnográfico (Álvarez Gandolfi, 2014, 2017) basado en técnicas de observación participante y de entrevistas abiertas en las instancias de socialización -presenciales y mediadas digitalmente- en las que participan los otakus, entendiendo que dicho abordaje puede enriquecer las reflexiones sobre la dataficación dela sociedad(Masson, 2017). En ese contexto, se seleccionaron específicamente los videos subidos al canal de YouTube de Kalathras, cuyo interés principal se centra en el animé y en los videojuegos -usualmente también asociados con los consumos de animaciones e historietas-. Su popularidad entre los fans iberoamericanos de objetos de la cultura de masas japonesa se verificó, además de en los criterios cuantitativos mencionados, en las recurrencias de las verbalizaciones de los otakus referidas a su youtuber de habla hispana preferido, en las que sobresalía Kakathras como creador de contenidos vinculados con los productos mediáticos nipones. 


\section{LA INTERFAZ DE YOUTUBE Y SUS POSIBILIDADES CONECTIVAS DE PARTICIPACIÓN}

José Van Dijck (2016) observa que, en el marco de una cultura de la conectividad, la producción de la socialidad y las conexiones humanas están mediadas por plataformas digitales, a las cuales no solo propone comprender como estructuras socioeconómicas, sino también como constructos tecnoculturales. Entre estas plataformas, YouTube se presenta desde 2005 como un medio conectivo con una promesa de participación que, al mismo tiempo, propaga la lógica comunicacional de la televisión tradicional y permite compartir videos de producción amateur que la desafían, dado su carácter alternativo o poco convencional. La apuesta de esta plataforma era - ¿y es?- convertir el consumo televisivo en una práctica interactiva alrededor de contenidos generados por los propios usuarios, aunque desde que fue comprada por Google en 2006, rápidamente empezó a colaborar con las industrias del entretenimiento. Tal cambio de orientación tuvo un correlato en la modificación de su interfaz, de los contenidos almacenados y de sus usuarios.

Cabe subrayar que YouTube presenta un diseño de interfaz relativamente amigable-es decir, sencillo e intuitivo- que, en términos teóricos, habilita distintos niveles de participación que van desde el consumo hasta la producción, pasando por la distribución y la socialización. A diferencia de lo que ocurría en 2008, cuando las características de la interfaz alentaban una participación activa y social de los usuarios - cristalizada en la centralidad que ocupaban los botones destacados para subir contenidos, comentar y hacer amigos (Van Dijck, 2016)-, en la actualidad los interpela como espectadores pasivos de videos (Burgess \& Green, 2018). De aquíque YouTubehaya pasado a adoptar el aspecto visual de la televisión y, en vez de presentarse como un depósito descentralizado de videos amateurs, tal cual lo hacía en sus inicios, empezó a mostrar una organización del contenido por canales según la lógica del narrowcasting, agrupados en categorías como "Música”, "Deportes", "Videojuegos", "Películas" o “Animación”. La gran mayoría de los videos disponibles en esta plataforma no recibe ningún tipo de valoración ni comentario, de lo que se infiere, a su vez, la predominancia de usuarios que se limitan a visualizar los contenidos (Gallardo Camacho \& Alonso, 2010). Dicha inferencia permite matizar, a priori, la consideración de que las audiencias en Internet siempre asumen, supuestamente, comportamientos productivos e interactivos.

Estas descripciones de la interfaz de la plataforma permiten, asimismo, un acercamiento a las formas en que pueden concretizarse los diferentes tipos ideales de usos que caracterizan a la cultura participativa. En virtud de los acelerados desarrollos tecnológicos responsables de la rápida obsolescencia de referencias a "viejos" y "nuevos medios" (Scolari, 2008), es necesario presentar una revisión actualizada y ampliada de los planteos sobre la plataforma y los usos de ella (Álvarez Gandolfi, 2018). Así, se advierte que, entre los usuarios 
de YouTube, en general, pueden reconocerse tres niveles graduales de participación: a) el del usuario periférico, que usa la plataforma para ver videos sin necesidad de registrarse; b) el del usuario miembro, que como está registrado en el sitio también puede valorar y comentar las obras audiovisuales con un "Me gusta" o un "No me gusta"; c) y el del usuario generador de contenidos, que abre y diseña un canal al cual los usuarios miembros pueden suscribirse para ver, comentar y valorar los videos que crea y/o sube allí.

Mientras que lo referido a la especificidad de los distintos tipos de uso que los otakus-en particular-pueden hacer de YouTube, aunque sin perder de vista los niveles de la categorización general de usuarios ni las motivaciones antes mencionadas, aquí se proponen otras tres conceptualizaciones, derivadas del trabajo de campo y las entrevistas cualitativas realizadas:

1) Uso expectante: consiste en buscar, desde la página principal de la plataforma, animaciones japonesas que estén disponibles para ver en el sitio. $\mathrm{Si}$ bien tampoco es necesario estar registrado para compartir los contenidos en otras páginas, la motivación que está detrás de este tipo de uso se asocia con la visualización, práctica que en sí misma contribuye a la popularidad de los videos del canal consumido por el carácter cuantitativo de la propia lógica de funcionamiento de YouTube.

2) Uso convergente: consiste en registrarse en YouTube, obteniendo una cuenta de Google a partir de la cual iniciar sesión y acceder al sitio, para poder valorar y comentar los videos, así como guardarlos en listas de reproducción. Este tipo de uso también permite suscribirse a canales desde los cuales ver contenidos relacionados con los productos mediáticos nipones. Y si bien el registro en la plataforma también habilita a generar y subir videos en canales propios, la motivación que está detrás del uso convergente se ubica a mitad de camino entre la lógica del broadcasting, propia de los medios tradicionales de diseminación uno-a-muchos, y la lógica del networking, propia de los medios digitales de intercambios muchos-amuchos. En efecto, la mayoría de los otakus registrados tienden a restringir su participación en el sitio a las acciones de ver para valorar y comentar, a diferencia del siguiente tipo.

3) Uso fan: consiste en diseñar y sostener un canal en el cual subir con cierta frecuencia contenidos de terceros -asociados en el caso del otakismo con la industria del animé- o de creación propia vinculados con ellos, así como hacer transmisiones en directo para fomentar la interacción sincrónica con los suscriptores que -preferentemente- sean pares interesados en estos videos en tanto parte del mismo fandom otaku o comunidad de fans de objetos de la cultura de masas japonesa. 
La posibilidad de hacer un uso expectante de YouTube beneficia a las personas que, en Iberoamérica, desean consumir animé vía Internet, pues a diferencia de lo que ocurre con su circulación masiva en Japón, estos productos mediáticos cuentan con una relativamente escasa distribución a nivel regional por las vías tradicionales asociadas con las industrias televisivas y cinematográficas. El acceso online a contenidos específicos por demanda no solo es una novedad que presenta la plataforma respecto de la televisión, sino que también permite satisfacer necesidades de distribución en función de la lógica del compartir (Lange, 2014). En este sentido, puede concebirse que este tipo de uso es una práctica participativa que indica una proximidad con las animaciones niponas, en el sentido de que al nivel personal de cada usuario supone como mínimo un conocimiento del nombre del contenido buscado.

Tal proximidad es el motivo por el que, en el caso específico de la expresión en esta plataforma del fanatismo por objetos de la cultura de masas japonesa, conceptualizado como otakismo (Álvarez Gandolfi, 2014, 2017, 2018), no podría hablarse de un uso "periférico" planteando una extrapolación lineal del término correspondiente al primer nivel general de participación del usuario por fuera de este fandom, pues los otakus realizan búsquedas de contenidos dentro de la temática central de los bienes simbólicos nipones alrededor de los cuales se estructura su comunidad. Así, el uso expectante propuesto aquí para conceptualizar una de las formas que puede cobrar el otakismo en YouTube se vincula con una dimensión activa y colectiva dado que se ve motivada por la realización de un aporte al fandom otaku, el cual consiste en incrementar la cantidad de vistas o reproducciones de los videos alojados en la plataforma que estén relacionados con los productos mediáticos provenientes de Japón y, en consecuencia, contribuir a su popularidad y circulación, aumentando su visibilidad en el sitio en la medida en que de ese modo ayudan a configurar los algoritmos de recomendación.

La motivación de avanzar un nivel más en la participación activa y colectiva está detrás del uso convergente que, a diferencia del uso expectante, no solo contribuye a la popularidad de los contenidos en YouTube relacionados con el fandom otaku aumentando su número de vistas o reproducciones, sino que también implica valorarlos y participar de la sección o caja de comentarios para interactuar con otros usuarios pares. En este sentido, los otakus se valen de las funciones sociales de la plataforma (Carlón \& Scolari, 2009; Snickars \& Vondereau, 2009; Fernández, 2016; Van Dijck, 2016; Burgess \& Green, 2018) para compartir informaciones, saberes, impresiones, opiniones, inquietudes, interpretaciones, consultas y/o sentimientos alrededor de sus consumos en común.

Por último, la participación implicada en el uso fan que puede asociarse con el otakismo en YouTube trasciende la supuesta pasividad prefigurada en la instancia de la recepción, según la lógica del broadcasting de los medios tra- 
dicionales, y, a diferencia del uso convergente, sus motivaciones van más allá del valorar, el comentar y el compartir, acciones propias de los medios digitales e interactivos regidos por la lógica del networking. De este modo, en este nivel se asume un mayor compromiso con los bienes simbólicos japoneses que lleva a querer abrir canales para difundirlos y/o realizar producciones propias a partir de sus consumos, mayor compromiso y productividad que son uno de los tantos rasgos por los que las personas pueden concebirse a sí mismas o ser concebidas por los demás como fans (Borda, 2012).

Por esto no es extraño que una de las manifestaciones del fanatismo en general dentro de esta plataforma sea valerse de ella para satisfacer necesidades de distribución, producción y expresión (Lange, 2014), con videos cuyo origen puede ser comercial, gubernamental, institucional, académico, independiente, profesional, sin fines de lucro o amateur, lo que ha llevado a algunos autores a pensar en YouTube como un espacio mediático híbrido (Varela, 2009; Burgess \& Green, 2018). Pero más allá de que pareciera que, en un contexto de convergencia, YouTube cristaliza la posibilidad de participación activa de múltiples receptores que pueden poner en circulación sus propias emisiones discursivas -mediante el intercambio de comentarios escritos o de contenidos audiovisuales misceláneos-, es preciso advertir las limitaciones de esa circulación, que pasa a ser centralizada dentro de la propia plataforma donde queda absorbida la heterogeneidad. Como ya lo advirtió Mirta Varela (2009) hace más de una década:

\footnotetext{
La imagen de la red que se impuso para representar las relaciones en Internet, se opone completamente al sistema de broadcasting en cuanto a la relación entre un emisor centralizado y muchos receptores. (...) En ese sentido, el lema de YouTube -broadcast yourself-viene a tocar un punto crítico del sistema. Saca partida de lo que se presentó como característica distintiva y saliente de Internet. Lo hace, sin embargo, a través de un sitio que absorbe la heterogeneidad en un sello único: todo pasa a través de YouTube (...) No se trata de una centralización de la emisión porque allí circulan videos de origen muy diverso: se trata de una centralización de la circulación (pp. 216 y 217).
}

Dicha centralización de la circulación consiste en que, si bien los usuarios pueden compartir los videos subidos al sitio haciéndolos circular por fuera de allí e incrustándolos otras plataformas -como por ejemplo Facebook-, las recomendaciones hechas por YouTube -de videos, canales o listas de reproducción- se generan tanto en función de la popularidad de los contenidos en este sitio como en función de su relevancia para cada usuario.

Por un lado, se contempla el procesamiento de la cantidad de visualizaciones, reacciones y comentarios que tienen los videos en toda la plataforma. Por otrolado, dichas recomendaciones coinciden con una traducción en metadatos de los registros del historial de búsquedas, reproducciones, suscripciones, lis- 
tados, valoraciones y publicaciones que los usuarios realizan en la plataforma y que, a su vez, están vinculados con cookies y actividades asociadas con las direcciones de IP desde las que navegan. Recomendaciones que, en consonancia con las lógicas de los algoritmos propias de la dataficación social (Livingstone, 2018), ya no les son solo sugeridas a los usuarios registrados en la plataforma, sino que aparecen ni bien se accede a la página principal de YouTube como usuario general, independientemente de haber accedido o no luego de iniciar sesión en una cuenta de Google.

A partir de tales algoritmos, YouTube sugiere automáticamente contenidos similares (Snickars \& Vonderau, 2009; Van Dijck, 2016; Burgess \& Green, 2018), de modo que los usuarios pueden llegar a no tener acceso a lo que quede por fuera de dichas variables personalizadas según sus consumos, gustos e intereses específicos. Si se extrapolan los planteos de Rafael Grohmann (2019), pareciera que, por más que los contenidos audiovisuales disponibles en la plataforma sean de acceso público y puedan llegar a tener un alcance masivo, las recomendaciones automáticas del sitio orientan los consumos prefigurando audiencias particulares con las que puedan relacionarse las navegaciones de cada usuario. O, como dirían Diego García Ramírez \& Dune Valle Jiménez (2020), los algoritmos computacionales moldean comportamientos y prácticas cotidianas de consumo a partir de manipulaciones opacas.

Hasta ahora se repensaron los niveles generales de participación en YouTube para reflexionar específicamente sobre distintos tipos de uso del sitio que, según se argumentó, pueden ser relacionados con diferentes motivaciones del otakismo. En el siguiente apartado se analizará cómo estos usos están atravesados por dinámicas colectivas de construcción identitaria y de expresión de sentidos de pertenencia al fandom otaku, que tiñen la apropiación social que tales fans hacen de dicha plataforma digital.

\section{FANIFICACIÓN Y LIIMITES DEL OTAKISMO EN YOUTUBE}

Llegado este punto, es posible adentrarse en la circulación de sentidos dentro de la plataforma. ¿De cuáles modos sus usuarios pueden ser identificados como otakus? ¿Cómo se apropian estos fans de ella? Así como en YouTube existen distintos niveles generales y graduales de participación, también existen diversas prácticas específicas de significación y de apropiación que se ven atravesadas por las dinámicas procesuales del otakismo, como fenómeno de construcción de identidades y configuración de comunidades. Y aquí cabe destacar una diferenciación entre las conceptualizaciones que movilizan los usos y las apropiaciones (Lago Martínez, Méndez \& Gendler, 2017), que se dan en un entramado de interacciones desiguales entre artefactos y usuarios que son la base de la cultura digital (Crovi Druetta, 2017). 
Si se hace una extrapolación adaptada de las perspectivas teóricas en torno de tales conceptos, podría pensarse que, mientras que los usos remiten a los objetos y a las características técnicas, las apropiaciones apuntan a los sujetos y a los sentidos de sus prácticas en diferentes contextos socioculturales e históricos. Es en el referido entramado dinámico que también podría sostenerse que el análisis de los vínculos que establecen los otakus entre ellos y con una plataforma como YouTube, con distintas motivaciones desde el punto de vista colectivo e individual, puede tomarse como un caso particular para comprender las distintas formas inclusivas y excluyentes que puede cobrar la participación en las sociedades contemporáneas hipermediatizadas.

Por lo tanto, es posible interpretar que las apropiaciones que hacen los otakus de YouTube implican su uso fan, es decir, aquel enmarcado en procesos de construcción identitaria y de pertenencia comunitaria, pero también su uso convergente y su uso expectante, dado que según se explicó en el apartado anterior estos también se ven atravesados por motivaciones enmarcadas en tales procesos. De este modo, dichos sujetos no solo se valen de esta plataforma para sortear restricciones de circulación y consumir contenidos que ponen en escena simbolismos diversos (Jenkins, 2009a), sino también para sentirse parte de un colectivo más amplio y explorar facetas creativas. Sin embargo, estas posibilidades también se ven afectadas por ciertas limitaciones y desigualdades que condicionan las apropiaciones que pueden hacer estos sujetos. Según se argumentará, de acuerdo con la tipología presentada por Silvia Lago Martínez, Anahí Méndezy Martín Gendler (2017), las apropiaciones predominantes vinculadas con el caso del otakismo en YouTube serían "reproductivas" y “adaptadas", pues usan la plataforma según los fines personales y ociosos del ver coincidentes con su desarrollo actual y, a lo sumo, apuntan a generar un uso distinto que vuelva a poner en un primer plano los fines colectivos y sociales del compartir entre pares, aunque de todos modos se encuentra condicionado por la lógica algorítmica.

Como se sugirió anteriormente, las posibilidades de participar en YouTube -como consumidor de videos que hace un uso expectante, suscriptor que deja likes y comentarios haciendo un uso convergente, difusor y/o creador de contenidos que hace un uso fan- están atravesadas por la convergencia entre medios tradicionales y digitales, la coexistencia de las lógicas de transmisión broadcasting y networking con las que respectivamente se asocian, y las prácticas socioculturales relacionadas con el fanatismo en general. Respecto de la expresión del otakismo en particular, cabe aclarar que las implicaciones identitarias de este panorama se manifiestan en distintas dimensiones que pueden ser leídas en función de ciertas lógicas comunitarias que atraviesan la apropiación social que hacen de dicha plataforma.

En principio, según se anticipó, el uso expectante de YouTube por parte de los otakus, que se corresponde con un primer nivel de participación general, 
consiste en buscar animaciones japonesas subidas a la plataforma o videos producidos por usuarios que refieran a ellas, para lo cual, si se pretende lograr resultados específicos, es necesario tener algunos de los códigos culturales vinculados con su universo simbólico y compartidos por el fandom, el grupo de consumidores habituales, al cual a su vez se contribuye en la medida en que la mayor visualización de tales contenidos aumenta su visibilidad en las recomendaciones automáticas del sitio. Esta dimensión colectiva también puede reconstruirse a partir de las manifestaciones personales del otakismo implicadas en el uso convergente y en el uso fan de la plataforma.

Manifestaciones que se corresponden con elementos expresivos que indican una pertenencia común entre usuarios consumidores, suscriptores y creadores que, como mínimo, tienen consumos e intereses compartidos: a) nicks, íconos de las cuentas o fotos de perfil e imágenes de portada para los canales, que refieren al otakismo y a los productos mediáticos japoneses sobre los que configura; b) presencia de frases o palabras japonesas en las interacciones que tienen lugar en la sección de comentarios o donde los difusores y/o creadores presentan sus videos e interpelan a sus eventuales espectadores; c) combinación de caracteres tipográficos o emojis utilizados como recursos que se vinculan con las expresiones de los personajes del animé. Estos son todos elementos que, a partir de las aproximaciones analíticas cualitativas señaladas al inicio de este trabajo, pudieron identificarse como recurrentes en la mayoría de las cuentas de usuario observadas desde las que se suben y/o comentan videos relacionados con los bienes simbólicos nipones.

Si bien las características actuales de la interfaz de YouTube parecieran estimular más el consumo individualizado que las prácticas de comunalización, cabe advertir que los fans de contenidos vinculados con objetos de la cultura de masas japonesa la fanifican u "otakizan" en un doble sentido: no solo se la apropian para construir y expresar una identidad otaku a nivel personal, sino que también la utilizan para poner en circulación sentidos de pertenencia colectiva. Así, sacan provecho de las funciones sociales del sitio aun pese a que, como observa Van Dijck (2016), en términos de diseño tales funciones vinculadas con el "compartir" hayan perdido visibilidad frente a las opciones vinculadas con el "ver". De aquí que no puede perderse de vista la importancia de la Sección de comentarios para la generación y reafirmación de lazos sociales entre fans, incluso aunque su carácter conversacional se vea dificultado por la asincronía y unidireccionalidad predominantes de las comunicaciones que suceden allí.

En este sentido, es posible concebir la participación de los usuarios a través de los "Me Gusta" o likes, los comentarios, las suscripciones y la opción de compartir los videos en otros sitios como promotora de actividades grupales, de modo que estas funcionalidades son indicadores culturales que permiten inferir una alineación con los valores de comunidades de gusto conformadas alrededor de videos compartidos sobre un tema determinado (Burgess \& Green, 
2018). Tema que, en el caso de los otakus, involucra a los objetos de la cultura de masas japonesa. Y participación que, en el ánimo de la centralización de la circulación dentro de los límites de YouTube y sus algoritmos personalizados (Varela, 2009; Van Dijck, 2016), termina por aumentar la visibilidad de aquello que coincide con los propios valores, gustos e intereses. Pero tales metadatos de perfil no solo conectan contenidos a usuarios específicos por afinidad, sino que también habilitan las conexiones entre consumidores: aquí reside una de las dimensiones por las cuales puede pensarse quelos otakus realizan mayoritariamente unas apropiaciones reproductivas y adaptadas, aplicando la tipología ya citada (Lago Martínez, Méndez \& Gendler, 2017), pues si bien la lógica algorítmica limita la circulación restringiendo las recomendaciones a los contenidos vinculados con bienes simbólicos nipones, al mismo tiempo habilita que sus fans se conecten y accedan a nuevos videos en el marco del otakismo.

Tanto a partir de los elementos expresivos señalados como sobre la base de sus intercambios en YouTube, los otakus se fanifican a sí mismos, en el sentido de que se presentan de modo tal que puedan ser identificados como un par fan por parte de un colectivo de fans con consumos e intereses compartidos. A su vez, esta fanificación individual se constituye como una base para fanificar a otros usuarios que compartan y reconozcan los códigos culturales vinculados con el otakismo. Los fans de objetos de la cultura de masas japonesa pueden valerse de la lógica del networking de los medios digitales e interactivos para configurar y sostener una identidad grupal entre usuarios, poniendo en juego sus códigos compartidos y relacionados con las prácticas de consumo de las animaciones niponas.

Aquí cabe recordar que, en YouTube, las cuentas individuales de quienes hacen un uso fan de la plataforma pasan a configurarse como canales en los que se sube animé o videos relacionados con la temática, apuntando al reconocimiento de un colectivo de pares que buscan ese tipo de contenidos. Esto se debe a que puede pensarse que se transforman en medios de comunicación broadcasting para paliar la falta de circulación del animé en las grillas televisivas de programación o en las salas de cine a nivel regional por fuera de Japón.

En este último sentido, a diferencia de lo que sostienen los planteos generales de Burgess \& Green (2018) sobre los usos generales de la plataforma, es posible entender que, en su caso específico, los otakus se apropian de YouTube para eludir los canales de distribución de las industrias del entretenimiento dominantes. Asimismo, la visibilidad más pública de los contenidos alojados allí pareciera ofrecer mejores posibilidades para una autocomunicación de masas (Castells, 2009), de modo tal que, al mismo tiempo que habilita una mayor circulación de las significaciones vinculadas con el otakismo, implica la necesidad de definir los destinatarios de los mensajes que se generan contemplando que pueden llegar potencialmente a una audiencia global, por lo que podría pensarse que las intervenciones en este sitio tienden a presentar un 
fuerte carácter comunalizador en comparación con los intercambios en otros medios digitales.

No obstante, si bien los otakus pueden apropiarse de YouTube para eludir los canales dominantes de distribución, esto no significa que todos los contenidos relacionados con el fanatismo por objetos de la cultura de masas japonesa y alojados en tal medio digital tenga los mismos significados y valores para sus espectadores, así como tampoco quiere decir que dichos videos puedan ser igualmente "populares". Entonces, siguiendo los criterios de popularidad que hacen a la youtubeidad o cultura común de la plataforma (Burgess \& Green, 2018), a continuación se analizarán los vínculos que propone uno de los youtubers de animé más populares entre los otakus para relevar cómo construye un colectivo a partir de su posicionamiento enunciativo.

\section{KALATHRAS, THE ANIME GUY. O CÓMO PUEDE CONSTRUIRSE UN COLECTIVO HIPERMEDIÁTICO ALREDEDOR DE UN ENUNCIADOR INDIVIDUAL}

Aunque algunos planteos sobre YouTube en general (Burgess \& Green, 2018) coinciden en que los contenidos audiovisuales generados por profesionales de las industrias culturales, asociados a los medios tradicionales, tienden a ser los "videos más vistos" y marcados como favoritos o añadidos a los perfiles personales de los usuarios, en el caso del otakismo no ocurre lo mismo. Aquellos videos vinculables con los objetos de la cultura de masas japonesa que tienen mayor número de reproducciones, valoraciones y comentarios no son los que reproducen animaciones niponas de producción industrial, sino los que refieren a otro tipo de contenidos relacionados con ellas creados por los usuarios: por ejemplo, entradas de video o vlogs testimoniales, donde los otakus muestran sus reacciones o expresan sus opiniones ante distintos animé o ante la sociedad y la cultura japonesa en general. También videos de habilidades artísticas, entre los que se destacan covers vocales o instrumentales de openings y endings -es decir, canciones que dan inicio y cierre a tales contenidos-, pasos de baile, performances de cosplay o caracterizaciones interpretativas de personajes que incluyen vestuarios y accesorios, Anime Music Videos [AMV] o ediciones de distintas escenas musicalizadas con canciones diferentes a las de los textos fuente, a los que se suman diferentes tipos de tops clasificatorios y recomendatorios de géneros u obras, como "los 10 mejores animé de drama/romance/comedia". Estos fans prefieren consumir animé vía Internet no por esta plataforma, sino en sitios web específicos que garantizan mejor calidad y la no censura de las connotaciones sexuales y violentas con las que suelen asociarse diversos títulos -que infringirían las condiciones de uso de la plataforma para subir videos, así como derechos de autor-, acudiendo a YouTube para visualizar contenidos fan derivados de estas animaciones. 
Por otro lado, como se advirtió, lo que ocurre es que tampoco todos estos contenidos cobran los mismos sentidos ni apreciaciones, sino que se aplican criterios según los cuales unos logran predominancia por sobre otros, tanto simbólica como material. En este sentido, resulta productivo pensar en el nivel de los usuarios generadores de contenidos -youtubers- que se han unido al Programa de Socios o Partners de YouTube para monetizar su participación allí mediante anuncios.

En efecto, es posible pensar que, a partir de su fanificación individual, tales usuarios se constituyen como enunciadores hipermediáticos capaces de generar su propio colectivo (Fraticelli, 2019) de fans entre pares, alrededor de su figura. Estos otakus logran que otros otakus se suscriban a sus canales y consuman los contenidos audiovisuales que suben allí, como lo es el caso del youtuber otaku Kalathras. Para reflexionar sobre su figura como parte de los "nuevos sujetos mediáticos" (Scolari \& Fraticelli, 2017) que surgen a caballo de las plataformas digitales e interactivas, pueden retomarse las apropiaciones que Mario Carlón (2017) hace de los planteos comunicacionales veronianos con el objetivo de repensar las complejidades de la circulación del sentido en las sociedades contemporáneas hipermediatizadas. De este modo, es posible contemplar los modos en que la producción y el reconocimiento constituyen instancias de enunciación que distintos actores pueden ocupar de modo intercambiable-medios e instituciones, individuos y colectivos-, así como las diferentes direcciones complejizadas -ascendentes y horizontales, pero también descendentes y verticales- que puede seguir la comunicación.

No solo los fans pueden visibilizar sus discursos de modo público, sino que también pueden generar colectivos, posibilidad antes reservada a las instituciones y los medios tradicionales de comunicación masiva. De aquí que resulta enriquecedor analíticamente considerar los vínculos que los enunciadores hipermediáticos proponen con sus espectadores para relevar cómo construyen un colectivo. En el caso de Eric Cuaresma, más conocido como Kalathras, podría aplicarse la categorización planteada en el segundo apartado de este artículo en pos de analizar cómo el uso fan que este sujeto hace de YouTube a su vez conduce a una fanificación de los otakus que consumen sus contenidos relacionados con objetos de la cultura de masas japonesa. Y, según se explicará, la participación implicada en esta fanificación se restringe a un uso convergente, estimulado entre sus suscriptores por Kalathras, y mayoritariamente a un uso expectante, que contrasta con el promocionado por dicho enunciador, aunque de todos modos contribuya a su popularidad al conllevar un aumento de las visualizaciones de sus videos.

Este youtuber español es considerado por la mayoría de los fans iberoamericanos de las animaciones japonesas como uno de los mejores. En un nivel global, su éxito se corresponde con los criterios cuantitativos de popularidad en YouTube antes mencionados: su canal, Lolweapon, cuenta con 1.78 millo- 
nes de suscriptores a julio de 2021. En sus comienzos, desde el 29 de marzo de 2012, subía gameplays, es decir, contenidos vinculados a videojuegos como Darksiders, Torchlight, Dishonored y Metal Slug. Casi un año más tarde, desde el 15 de enero de 2013, comenzó a subir videos de reseñas, reacciones y tops de animé, momento en el cual se observa una especie de salto en su popularidad: pasó de un promedio de 5.000 visualizaciones, calculado entre los veintitrés videos de gameplays que subió durante su primer año en YouTube, a un promedio de 325.000 visualizaciones, calculado entre sus diez primeros videos de reseñas, reacciones y tops de animé. En la actualidad, los diez videos más vistos de su canal tienen entre 1.8 millones y 4.4 millones de visualizaciones. Contrastado con la oferta de videos disponibles en otros canales de YouTube también consumidos por los otakus y relacionados con los objetos de su fanatismo, según pudo relevarse a partir del doble trabajo de campo ya referido, su principal diferencia no consiste en su especialización temática -pues como se señaló hay una gran cantidad de contenidos basados en animaciones japonesas creados por usuarios como Kalathras, que se centran en reacciones, opiniones, reseñas, tops, análisis y noticias sobre animé-, sino más bien en su estilo y sus posicionamientos enunciativos.

Uno de los posibles caminos para comprender las razones por las cuales en un año aumentaron las visualizaciones de los videos de Kalathras en un $6.500 \%$, para luego pasar de un promedio de 325.000 a un promedio de 2.250 .000 calculado entre los diez videos más vistos de su canal en la actualidad, consiste en analizar los procedimientos discursivos que juegan en la construcción de este youtuber como nuevo enunciador hipermediático individual. En principio, tanto su foto de perfil como la cabecera de su canal se corresponden con un uso fan que hace de la plataforma, a partir de un diseño que indica su pertenencia al fandom otaku, lo que se refuerza con su presentación como The Anime Guy -El chico animé-: en la primera foto puede vérselo en primer plano con un cosplay de Kamina, personaje de Tengen Toppa Gurren-Lagann-pelo teñido de turquesa con flequillo largo hacia la derecha, lentes triangulares con un filtro naranja y una capa roja-; mientras que en la segunda cabecera puede verse la bandera del Sol Naciente en el fondo, personajes protagonistas de distintas animaciones japonesas -como Naruto, Saint Seiya, One Piece e Inuyasha- y el nombre del canal centrado por delante.

Si se retoman los planteos de Carlos Scolari \& Damián Fraticelli (2017), cabe destacar dos tipos de operaciones semióticas predominantes en los contenidos audiovisuales de Kalathras -y de los youtubers en general-, que conforman sus gramáticas productivas. En primer lugar, se observa una enunciación edificada en el contacto, es decir, en términos jakobsonianos, sus videos presentan una acentuada función fática que reafirma el vínculo con sus enunciatarios mediante recursos de naturaleza discursiva -semejantes a los de la televisión y el cine- y de dispositivo mediático - propios de la plataforma-. En el caso de 
este youtuber en particular, prima su habla constante como marca representativa: en sus tops, reacciones, reseñas, rankings comparativos, recomendaciones, noticias, videos de curiosidades y estrenos, su cuerpo tiende a no aparecer en cámara, pero su voz en off no deja de escucharse.

Asimismo, en segundo lugar, vinculada con esta habla constante, se observa una enunciación edificada en la afectividad, es decir, sus videos presentan una acentuada función emotiva, nuevamente en términos jakobsonianos, que resalta el estilo individual del youtuber, el carácter no institucional de su producción. En sus contenidos audiovisuales, Kalathras saluda y da la bienvenida a todos sus espectadores - a quienes apela continuamente-, reflexiona sobrelas temáticas que trata y cuenta las sensaciones que les generan intercalando tanto un tono serio y humorístico como un lenguaje formal y coloquial, así como aclara con especial hincapié que las clasificaciones dependen de sus gustos propios y preferencias personales. De este modo, su uso fan personalizado de la plataforma se relaciona con la promoción de un uso convergente entre sus consumidores dado que implica un llamado a la (re)acción, la suscripción y el comentario, que se basa en la lógica comunitaria del compartir referencias entre pares y los contenidos que las contienen en otros sitios, acciones que contribuyen a su mayor popularidad.

Tales observaciones pueden complementarse con las ideas desarrolladas por Damián Fraticelli (2019) para contribuir ala comprensión delas cualidades y los modos en que operan los colectivos mediáticos (Carlón, 2016a) en el nuevo sistema de los medios digitales con base en redes sociales e Internet. Retomando las acepciones veronianas de "colectivo", se subraya la diferenciación entre colectivos de identificación y colectivos de comunicación: los primeros son una clase de actores que una sociedad reconoce como tal, identificándolos a largo plazo bajo una determinada nominación y otorgándole cualidades o comportamientos específicos; los segundos están formados por individuos heterogéneos que se agrupan en torno de un foco de interés común que concentra su atención, produciendo un vínculo social acotado.

A su vez, siguiendo las reformulaciones que Carlón propone de tales planteos veronianos, Fraticelli destaca que en la sociedad hipermediatizada contemporánea ya no solo las instituciones y los medios en instancia de producción generan discursividad siendo capaces de construir colectivos, sino que los colectivos e individuos antes ubicados en instancia de reconocimiento también pueden hacerlo. En este punto, el autor señala que una de las diferencias entre los colectivos mediáticos conformados por los medios masivos ylos de las redes sociales es la individuación de sus integrantes. Dicho señalamiento se relaciona con los recursos de dispositivo que indican la especificidad de los medios digitales como YouTube (Scolari \& Fraticelli, 2017): el contador de visualizaciones, de suscriptores, de $\mathrm{Me}$ gusta este video y No me gusta este video, el botón de compartir y la sección para añadircomentarios públicosasociadosal nickname del espectador quelos realiza. 
A partir de lo expuesto, puede afirmarse que, a partir de su uso fan de YouTube, Kalathras se constituye como enunciador individual que produce discursividad y genera un colectivo hipermediático asumiendo un posicionamiento profesional, expresado en sus amplios conocimientos de animé que le permiten cubrir en susvideos una gran variedad degénerosytítulos, trascendiendolos más conocidos. Al mismo tiempo, ¿y paradójicamente?, este colectivo se construye a través de una dirección comunicacional horizontal, de intercambios entre pares -enunciadores de igual estatuto en tanto que comparten el mismo fanatismo por las animaciones japonesas- con los que se establece cierto vínculo simétrico.

En sus inicios, este youtuber publicaba videos en los que abría votaciones para determinar los próximos títulos de animé a reseñar y contemplaba los comentarios de los espectadores que los realizaban asumiendo la postura enunciativa de los fans, en una dinámica de mutuo condicionamiento con su colectivo por la que el reconocimiento se convierte en parte de las condiciones de producción ${ }^{2}$. No obstante, las opciones entre las cuales podía elegirse eran seleccionadas por Kalathras, quien justificaba los motivos por los cuales dejaba afuera ciertos pedidos de modo inmediato, tranquilizando a sus espectadores al aclararles que posteriormente sí iba a reseñarlos. Dicha selección previa limitaba en sí misma las posibilidades del uso convergente alentado por este youtuber entre sus fans.

Ahora bien, centrarse en este tipo de análisis de sus videos no permite dar cuenta del crecimiento exponencial de sus visualizaciones. Para ello, hay que contemplar que el salto de popularidad de Kalathras en YouTube se vincula con las dimensiones que se desarrollarán a continuación. En principio, se generó una expansión de su colectivo al transformarse su uso fan y su posición como enunciador: como se anticipó, pasó de centrarse en subir a su canal gameplays en 2013 a centrarse en subir contenidos relacionados con el animé en $2014^{3}$ cambiando las categorías bajo las cuales publica sus contenidos en la plataforma -de "Videojuegos" a "Cine y animación"-, y ampliando así sus potenciales enunciatarios ${ }^{4}$ al mismo tiempo que comenzó a subir videos de modo más continuado.

2 En otros términos, podría pensarse que tal dinámica responde al funcionamiento de YouTube como zona de contacto (Fausto Neto, 2010) a partir de protocolos de interacción mediante los cuales se acciona la actividad discursiva de los espectadores pidiendo su colaboración e invitándolos a participar de las votaciones, lo que contribuye a la construcción del colectivo. Para Fraticelli (2019), a su vez, esto se condice con otra de las especificidades de los colectivos hipermediáticos: la interacción entre ellos y los enunciadores signada por la posibilidad de albergar sus reconocimientos con escaso desfasaje temporal y baja mediación institucional.

3 Cabe destacar que, en 2014, abrió doscanales más: uno también dedicadoal animéy muy similar a su canal principal (Kalathras Zone, con 794 mil suscriptores a la fecha), y otro centrado en los videojuegos (Kalathras Fantasy VIII, con 474 mil suscriptores). Dado que tanto en términos cuantitativos como cualitativos se entiende que su canal principal, Lolweapon, es el más representativo -como señalan la mayoría de los otakus y según puede advertirse en función de su considerablemente mayor número de suscriptores, visualizaciones y comentarios-, se optó por focalizar el análisis a partir de aquel. Queda pendiente para futuros trabajos un abordaje comparativo.

4 Según Jenkins (2008), el cine y la animación japonesa atraen a un público más diverso en comparación con los videojuegos. Esto se condice con la conformación del fandom otaku a nivel nacional, que tiende a preferir el animé por sobre otros consumos asociados con la identidad gamer (Álvarez Gandolfi, 2017). 
Dicha transformación, asociada con el cambio de su estatuto, se vio acompañada por la aparición en su canal de otro tipo de videos que incrementaron el uso convergente y su valorización por parte de su colectivo: especiales para celebrar el haber alcanzado 100.000 suscriptores el 30 de noviembre de 2014, agradeciéndoles a todos por seguir sus videos y reconociéndolos como parte indispensable del crecimiento de su canal, lo que puede interpretarse como un procedimiento para atenuar la asimetría que atraviesa su posicionamiento como experto y así sostener el pacto enunciativo amateur de igualdad; $\mathrm{y}^{\mathbf{5}} v \operatorname{logs}$ sobre su vida ${ }^{6}$.

A su vez, por fuera -pero también por dentro- de YouTube, cabe destacar su nombramiento en 2014 como "embajador" del ya mencionado Crunchyroll, sitio de pago de transmisión por streaming con más de 900 títulos de animé licenciados, lo que le permitió promover sus contenidos y obtener beneficios a cambio de publicitar dicha plataforma entre sus espectadores, alentando en este caso un uso expectante limitado a la visualización y al consumo; y sus participaciones en 2015 en distintos eventos para otakus como los que tuvieron lugar en Barcelona y en Buenos Aires, a los cuales fue invitado por pedido de sus seguidores a las empresas organizadoras -Ficomic y Jigoku Producciones, respectivamente-, habilitando vínculos no mediatizados entre el enunciador y un parte de su colectivo hipermediático, participaciones que también juegan en la expansión de este último en la medida en que Kalathras subió a su canal los videos que creó en referencia a ellas.

Por otro lado, en el marco de cierta relación intersistémica -entendiendo que esta consiste "en apropiaciones que las redes realizan de contenidos de los medios masivos o al revés" (Carlón, 2017, p. 14)-, el crecimiento de su popularidad en YouTube también se relaciona con su participación en los medios masivos: dos videos que datan del 28 de septiembre de 2009 fueron encontrados por sus seguidores entre 2014 y 2015 , quienes se valieron de las posibilidades del uso convergente para comentarlos preguntándose si era él o compartiendo su descubrimiento, así como expresando apoyo a sus dichos. Se trata de su aparición en el magazine Espejo Público, emitido por Antena 3, donde lo entrevistan en el marco de un informe sobre los otakus -tras conocerse que la hija del entonces presidente José Luis Rodríguez Zapatero era parte del fandom-como una "nueva tribu urbana", categoría que continúa usándose para categorizarlos

5 “Mis 10 Animes Favoritos / Especial 100.000 Subs!" cuenta con 1.546.810 visualizaciones a la fecha y, como advierte Kalathras, era un contenido muy ansiado por sus seguidores, quienes no solo esperan de él información compartida con su estilo humorístico, sino también recomendaciones, en la medida en que valoran sus opiniones que, como se mencionó anteriormente, el youtuber destaca que son exclusivamente personales. A su vez, en este tipo de videos aparece en su habitación empapelada con posters de animé mirando a cámara -otro recurso de naturaleza discursiva que acentúa la función fática-, algo que le es muy solicitado por sus fans y que no es tan habitual en su canal, de modo que puede leerse como una suerte de "regalo" para ellos y como ejemplo de que el reconocimiento constituye sus condiciones productivas.

6 El 7 de febrero de 2016, subió el video “50 COSAS SOBREMI|Kalathras”, el primero de este tipo en su canal, lo que puede interpretarse como un momento en el que exterioriza su vida privada hacia su colectivo, quizás a modo de agradecimiento por su creciente popularidad. 
a pesar de que estos fans la rechazan tanto entre ellos como en sus apariciones mediáticas, pues entienden que su fanatismo "no es pasajero".

Frente a las constantes representaciones mediáticas estereotipadoras ${ }^{7}$, Kalathras se posiciona en dichas apariciones en los medios masivos como enunciador polémico que las cuestiona: aparece luciendo vestimentas propias del visual $\mathrm{kei}^{\mathbf{8}} \mathrm{y}$, ante las típicas preguntas de extrañamiento que se realizan y muchas veces se traducen en reprobación - ¿¿Salen a la calle disfrazados? ¿Van así al instituto? ¿Trabajan?”-, resalta el marco situado de la práctica, reservada a momentos específicos como los eventos temáticos de animé en los que estos fans se encuentran presencialmente para compartir distintas actividades. Esto se complementa con su participación en entrevistas para medios digitales, como el canal de YouTube AwesomenessTV ES -en 2016-, en las que destaca los valores positivos de "la diferencia" con la que se identifican los otakus, lo que explica su interés por la diversidad cultural de las costumbres japonesas a cuya representación acceden mediante el animé. En todos estos casos, el youtuber apela al discurso alternativo del fandom otaku -curiosamente coincidente con el relato y el contrato fundacional de YouTube (Carlón, 2016b)-, entablando luchas por la significación en torno de las categorizaciones peyorativas del fandom otaku, indicando su pertenencia a él, generando identificación entre pares y estableciendo un vínculo simétrico con ellos. Si bien podría pensarse que este posicionamiento se asume conscientemente para aumentar visualizaciones e ingresos según los mecanismos de monetización de la plataforma, también es cierto que la participación fanificada de Kalathras allí data de hace casi una década, cuando todavía el fandom otaku no era tan visible y era todavía más estigmatizado en función de su ética de la alternatividad por fuera de Japón (Eng, 2012; Álvarez Gandolfi, 2014). En efecto, una de las estrategias más habituales de estos fans para hacer frente a los estereotipos que aún pesan sobre ellos consiste en reivindicar su identidad otaku, significada en términos de "rareza" o "anormalidad", como emblema de orgullo.

Por lo tanto, es posible afirmar que el colectivo que construye Kalathras depende dela activación de otro colectivo de identificación previoy más amplio generado a partir de los medios masivos: el de los otakus, fans de las animaciones japonesas, transmitidas tanto por la televisión como por el cine y a las que

7 Los núcleos discursivos de la "peligrosidad" y la "ridiculez" que atraviesan estas representaciones fueron reconstruidos en otro trabajo (Álvarez Gandolfi, 2017). En la nota de Antena 3 antes referida se expresan mediante risas de la conductora y los panelistas porque "no visten normal", "van a todos lados disfrazados" y "no quieren renunciar a su identidad infantil"; graphs que hablan de "adoración" vinculando estos consumos con una suerte de "obsesión" propia del fanatismo religioso; advertencias del "profesor especialista" invitado al panel asociadas con cierta preocupación porque "se refugian en la ficción" y le dedican una "excesiva cantidad de tiempo"; y críticas expresadas mediante mensajes de texto que mandan los telespectadores a la producción y van apareciendo en pantalla, como por ejemplo "un pico y una pala le daba to a esos niños" o "aparezco vestido así en mi pueblo y me destierran".

8 Movimiento estético cercanoal cosplayen tanto que caracterización mediante vestimentasy accesorios específicos inspiradas en el estilo de los personajes de animé que en cierta medida influyen en el estilo andrógino delos músicos japoneses de glam rock y metal gótico. 
actualmente se accede de modo mayoritario por diversos sitios de Internet. Las constantes referencias presentes en las producciones discursivas de Kalathras a temáticas y saberes valorizados por tal fandom permiten pensar que ese colectivo mediático es "madre" del colectivo hipermediático de este youtuber. Y es valiéndose de esas referencias que este sujeto estimula un uso convergente de la plataforma entre sus suscriptores.

El hecho de contemplar estas dimensiones podría dar cuenta de la posibilidad de construir un colectivo expandido entre pares a partir de un uso fan que conlleva un posicionamiento como enunciador profesional con conocimientos expertos o especializados, atravesada por las tensiones del vínculo asimétrico que implica. Esto no resultaría tan paradójico en la medida en que, como observa Jenkins (2008), los fandoms forman comunidades de conocimientos en las que se valoriza a quien posee mayores saberes sobre el foco del fanatismo -el animé, en el caso de los otakus-ylos comparte con el resto, lo que en el caso de Kalathras se ve potenciado por su estilo individual humorístico.

Si bien los colectivos mediáticos no necesariamente implican la constitución de colectivos identitarios, podría pensarse que en esta oportunidad sí lo hacen: el vínculo no termina cuando termina un video, sino que se prolonga en los otros contenidos del youtuber, en otros videos con los que se lo asocie en la propia plataforma y en sus producciones en otras redes sociales como Facebook, Twitter e Instagram. Todo ello en el marco más general de la comunidad de fans del animé - de aquí los conocimientos previos que demanda el uso expectante-, a la que podría sostenerse que los espectadores de Kalathras -así como el propio youtuber- pertenecen, colectivos que forman parte de las condiciones productivas de sus videos.

Pero, por otrolado, no todos los otakus en YouTube alcanzan la popularidad de Kalathras, observación que resulta relevante en la medida en que aún se realizan planteos en cierta medida "celebratorios", como los del propio Jenkins antes citado, que tienden a olvidar las jerarquías y las asimetrías que persisten en el orden sociocultural y que se trasladan a los medios digitales. Jerarquías y asimetrías que, como se advirtió hasta aquí, atraviesan los vínculos del youtuber con su colectivo, tensionadas por una dirección comunicacional horizontal entre pares del mismo fandom otaku. De ello que sus posicionamientos como enunciador profesional y polémico le permiten ser reconocido por sus fans en razón de sus saberes y, al mismo tiempo, como un fan más.

\section{CONSIDERACIONES FINALES}

Frente a los cambios que atraviesan las sociedades contemporáneas hipermediatizadas en el marco de la digitalización, en este artículo se propuso repensar los modos de conceptualizar las prácticas fan a partir de un estudio de las formas que pueden cobrar las manifestaciones relacionadas con el ota- 
kismo en YouTube. Para ello se reflexionó sobre las posibilidades y los límites de las apropiaciones que los fans de objetos de la cultura de masas japonesas -como el animé- pueden hacer de esta plataforma, atravesadas por lógicas tanto personales como colectivas de fanificación y por dinámicas algorítmicas que orientan la participación allí, conduciendo a una conectividad en un doble sentido: entre contenidos similares que se vinculan con los propios gustos e intereses, por un lado, yentre otros usuarios que comparten esos mismos gustos e intereses, por otro.

Así se planteó una categorización de los distintos tipos específicos de uso expectante, convergente y fan que los otakus pueden hacer de YouTube en función de diferentes motivaciones, analizando sus relaciones con los niveles generales y graduales de participación en el sitio por fuera de este fandom, como usuario periférico, registrado y generador de contenidos.

Según lo advierte Carlón (2016b), "YouTube es tanto un síntoma del fin de los medios masivos como al origen de una nueva era" (p. 142), en la medida en que permite que otros enunciadores, individuales y colectivos tomen el poder en la circulación discursiva. Las sociedades contemporáneas hipermediatizadas no resultan de procesos de homogeneización de las ofertas, relaciones y prácticas sociales, sino que las multiplican. Y, para advertir las complejidades, tensiones, transformaciones recíprocas, divergencias y jerarquías, la problemática de la circulación constituye un campo fecundo, pensada como desfase o diferencia que se apoya en una asimetría entre polos disímiles con lógicas diferentes (Fausto Neto, 2010; Verón 2013), asimetría que persiste en los sistemas mediáticos y socioindividuales.

Esta es una de las claves delectura que permite comprender el éxito de casos como el del youtuber otaku Kalathras, explorado en este trabajo. En efecto, el enfoque atento a la complejidad del dinamismo constitutivo delas significaciones y de los procesos de apropiación e interacción de la cultura digital habilita la contemplación de sus tensiones. Como se argumentó, son estas tensiones las que están detrás de la construcción de un colectivo hipermediático alrededor de la figura de un enunciador individual, que a su vez activa otro colectivo mediático de identificación previo y más amplio configurado en torno de referencias al otakismo, sobre la base de un uso fan a partir del cual se alienta un uso convergente. Y si bien el mayoritario es el uso expectante, este también aporta al crecimiento de la comunidad dado que incrementa la popularidad en la plataforma de los contenidos asociados con sus consumos. Por ello se destacó que las apropiaciones predominantes que los otakus hacen de YouTube pueden ser pensadas al mismo tiempo como "reproductivas" y "adaptadas", extrapolando la tipología correspondientemente remitida (Lago Martínez, Méndez \& Gendler, 2017).

Asimismo, estas observaciones permiten matizar el peso otorgado a la supuesta igualación que se daría entre los usuarios de las plataformas digitales 
en un contexto de ampliación del acceso, por el cual se da por sentado que se despliegan interacciones democráticas y descentralizadas (Jenkins, boyd \& Ito 2016). Pareciera que el foco en la creatividad y en la colaboración, que serían propias de una cultura participativa montada sobre la base de la convergencia mediática, lleva a relativizar las jerarquías intracomunitarias y sus formas excluyentes, en la medida en que se presupone a los fandoms como colectivos abiertos a todos quienes compartan los mismos gustos e intereses.

Para finalizar, quedan pendientes líneas de investigación a futuro que profundicen sobre interrogantes entre los que pueden encontrarse aquellos referidos a las diferentes posturas en reconocimiento en torno de Kalathras -ancladas, por ejemplo, en clivajes etarios, de género y de clase-; la comparación de su desarrollo a lo largo del tiempo con el de otros youtubers también valorados en el fandom otaku; la figuración de estos actores individuales como enunciadores orgánicos; el papel de los dispositivos maquinísticos en la construcción de sus colectivos; las interpelaciones de estos creadores de contenido y los comportamientos de sus consumidores durante las transmisiones en directo; un abordaje que no solo contemple las interacciones entre los sujetos a través de las tecnologías digitales y condicionadas por ellas, sino también sus interacciones con ellas y más allá de ellas; la reconstrucción de los sentidos que estos fans atribuyen a las plataformas y a las competencias que requieren las prácticas de participación allí; y las propuestas enunciativas de otros videos alojados en YouTube o en otros sitios - como Twitch o Instagram- que también se encuentren vinculados con el otakismo aquí problematizado.

\section{REFERENCIAS}

Álvarez Gandolfi, F. (2014). Subcultura otaku. Tesina, Licenciatura en Ciencias de la Comunicación, Facultad de Ciencias Sociales, Universidad de Buenos Aires, Argentina.

Álvarez Gandolfi, F. (2017). Fanatismos contemporáneos y cultura de la convergencia. Tesis de Maestría en Comunicación y Cultura, Facultad de Ciencias Sociales, Universidad de Buenos Aires, Argentina.

Álvarez Gandolfi, F. (2018).Elotakismo en YouTube. Reflexiones sobre sus posibilidades identitarias y límites socioculturales. Unidad Sociológica, 3(11), 86-99.

Benítez Larghi, S., Lemus, M., Moguillansky, M. \& Welschinger Lascano, N. (2014). Más allá del tecnologicismo, más acá del miserabilismo digital. Ensambles, 1, 57-81.

Bollmer, G. (2015). Technological Materiality and Assumptions About “Active” Human Agency. Digital Culture and Society, 1(1), 95-110. 
Booth, P. (2015). Playing Fans. Negotiating Fandom and Media in the Digital Age. Iowa: University of Iowa Press.

Borda, L. (2012). Bettymaníacos, luzmarianas y mompirris. El fanatismo en los foros de telenovelas latinoamericanas. Tesis de Doctorado, Facultad de Ciencias Sociales, Universidad de Buenos Aires, Argentina.

Burgess, J. \& Green, J. (2018). YouTube. Online Video and Participatory Culture. Cambridge: Polity Press.

Carlón, M. \& Scolari, C. (eds.) (2009). El fin de los medios masivos. El comienzo de un debate. Buenos Aires: La Crujía.

Carlón, M. (2016a). Apropiación contemporánea de la teoría de la comunicación de Eliseo Verón. En Vizer, E. \& Vidales, C. (coords.), Comunicación, campo(s) teorías yproblemas (pp. 125-153). Salamanca: Comunicación Social Ediciones.

Carlón, M. (2016b). Después del fin. Una perspectiva no antropocéntrica sobre el postcine, la post-tv y YouTube. Buenos Aires: La Crujía

Carlón, M. (2017). La cultura mediática contemporánea: otro motor, otra combustión (segunda apropiación de la teoría comunicacional de Eliseo Verón: la dimensión espacial). En Castro, P. (org.), Circulação discursiva. Entre produção e reconhecimiento (pp. 25-48). Maceio: Edufal.

Castells, M. (2009). La comunicación en la era digital. En Comunicación y poder (pp. 87-189). México: Siglo XXI.

Couldry, N. \& Hay, J. (2011). Rethinking Convergence/Culture. An Introduction. International Journal of Cultural Studies, 25(4), 473-486.

Crovi Druetta, D. (2017). Prácticas de apropiación e interacción en la cultura digital. En Cabello, R. \& López, A. (eds.), Contribuciones al estudio de procesos de apropiación de tecnologías (pp. 25-38). Rada Tilly: Ediciones Del Gato Gris.

Eng, L. (2012). Strategies of Engagement. Discovering, Defining, and Describing Otaku Culture in the United States. In Ito, M., Okabe, D. \& Tsuji, I. (eds.), Fandom Unbound. Otaku Culture in a Connected World (pp. 85-104). New Haven: Yale University Press.

Fausto Neto, A. (2010). A circulação alem das borda. Em Fausto Neto, A. \& Valdettaro, S. (dirs.), Mediatización, sociedad y sentido (pp. 2-17). Rosario: UNR editora.

Fernández, J. L. (2016). Plataformas mediáticas y niveles de análisis. InMediaciones de la Comunicación, 11(11), 71-96.

Fraticelli,D. (2019).Los colectivos mediáticos delas redes. Algunasobservaciones desde el humor ¿y más allá? InMediaciones de la Comunicación, 14(1), 47-63.

Gallardo Camacho, J. \& Alonso, A. (2010).Labaja interacción delespectador devídeosen Internet: caso Youtube España. Revista Latina de Comunicación Social, 65, 421-435. 
García Ramírez, D. \& Valle Jiménez, D. (2020). Los impactos de la ideología técnica y la cultura algorítmica en la sociedad: una aproximación crítica. Revista de Estudios Sociales, 71, 15-27.

Grohmann, R. (2019). Financeirização, midiatizaçãoe dataficação como síntesis sociais. InMediaciones de la Comunicación, 14(2), 97-117.

Hidalgo-Marí, T. \& Segarra-Saavedra, J. (2017). El fenómeno youtuber y su expansión transmedia. Análisis del empoderamiento juvenil en redes sociales. Fonseca. Journal of Communication, 15, 43-56.

Hills, M. (2013). Fiske's "textual productivity" and digital fandom. Web 2.0 democratization versus fan distinction? Participations. Journal of Audience \& Reception Studies, 10(1), 130-153.

Jenkins, H. (2008). Convergence Culture. La cultura de la convergencia de los medios de comunicación. Barcelona: Páidós.

Jenkins, H. (2009a). Fans, blogueros y videojuegos. La cultura de la colaboración. Barcelona: Paidós.

Jenkins, H. (2009b). What Happened Before YouTube. En Burgess, J. \& Green, J. (2018), YouTube. Online Videoand Participatory Culture (pp. 109-125). Cambridge: Polity Press.

Jenkins, H.(2013). Textual Poachers. Television Fans and Participatory Culture. Londres: Routledge.

Jenkins, H., boyd, d. \& Ito, M. (2016). Participatory Culture in a Networked Era. Cambridge: Polity Press.

LagoMartínez,S.,Méndez,A.\&Gendler,M.(2017).Teoría,debatesynuevasperspectivas sobre la apropiación de tecnologías digitales. En Cabello, R. \& López, A. (eds.), Contribuciones al estudio de procesos de apropiación de tecnologías (pp. 75-86). Rada Tilly: Ediciones Del Gato Gris.

Lange, P. (2014). Kids on YouTube. Technical Identities and Digital Literacies. Walnut Creek: Left Coast Press.

Livingstone, S. (2018). Audiences in an Age of Datafication:Critical Questions for Media Research. Television \& New Media, 20(2), 170-183.

Márquez, I. \& Ardèvol, E. (2018). Hegemonía y contrahegemonía en el fenómeno youtuber. Desacatos, 56, 34-49.

Masson, E. (2017). Humanistic Data Research. An Encounter between Epistemic Traditions. In Schäfer, M. T. \& van Es, K. (eds.), The Datafied Society. Studying Culture through Data (pp. 25-37). Amsterdam: AUP.

Scolari, C. (2008). Hipermediaciones. Elementos para una teoría digital interactiva. Barcelona: Gedisa. 
Scolari, C. \& Fraticelli, D. (2017). The case of the top Spanish YouTubers. Emerging media subjects and discourse practices in the new media ecology. Convergence, $35,51-63$

Snickars, P. \& Vonderau, P. (eds.) (2009). The YouTube Reader. Estocolmo: National Library of Sweden.

Van Dijck, J.(2016).Lacultura de la conectividad. Una historia crítica de las redes sociales. Buenos Aires: Siglo XXI.

Varela, M. (2009).Él miraba televisión, YouTube. La dinámica del cambio en los medios. En Carlón, M. \& Scolari, C. (eds.), El fin de los medios masivos, el comienzo de un debate (pp. 209-228). Buenos Aires: La Crujía.

Verón, E. (2013). La semiosis social 2. Ideas, momentos, interpretantes. Buenos Aires: Gedisa.

* Contribución: el trabajo fue íntegramente realizado por el autor.

* Nota: el Comité Editorial de la revista aprobó la publicación del artículo.

\section{(cc) BY}

Artículo publicado en acceso abierto bajo la Licencia Creative Commons - Attribution 4.0 International (CC BY 4.0).

\section{IDENTIFICACIÓN DEL AUTOR}

Federico Álvarez Gandolfi. Doctor (cand.) en Ciencias Socialesy Magíster en Comunicación y Cultura, Universidad de Buenos Aires (Argentina). Licenciado y Profesor en Ciencias de la Comunicación, Facultad de Ciencias Sociales, Universidad de Buenos Aires (Argentina). Becario, Consejo Nacional de Investigaciones Científicas y Técnicas (Argentina). Integrante, Núcleo de Estudios del Japón, Universidad Nacional deSan Martín (Argentina). Integrante, Comité Académico dela Red Iberoamericana de Investigadores en Animé y Manga. Sus intereses de investigación son las culturas fan, los medios digitales, los estudios de audiencia y los consumos de productos mediático-culturales japoneses. 


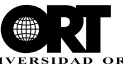

UNIVERSIDAD OR 